\title{
Local solvability of diagonal equations (again)
}

\author{
by \\ Christopher Skinner (Ann Arbor, MI)
}

1. Introduction. In this paper we return to the problem considered in $[\mathrm{B}]$ and $[\mathrm{S}]$, namely that of giving an upper bound on the integer $\Gamma(d)$, defined for each positive integer $d$ as the least integer such that any diagonal equation

$$
a_{1} x_{1}^{d}+\cdots+a_{s} x_{s}^{d}=0
$$

with coefficients $a_{i}$ in a $p$-adic field $K$ (i.e., a finite extension of $\mathbb{Q}_{p}$ ) has a solution $0 \neq\left(x_{1}, \ldots, x_{s}\right) \in K^{s}$ whenever $s>\Gamma(d)$ (that is, (1) has a non-trivial solution in $K$ ). Here and throughout, $p$ is taken to be a fixed prime. Of course, implicit in providing an upper bound on $\Gamma(d)$ is a proof of its existence!

Let $d=p^{\tau} m$ with $p \nmid m$. The main result of [B] asserts that

$$
\Gamma(d)<(2 \tau+3)^{d}\left(d_{1}^{2} d\right)^{d-1}, \quad d_{1}=(d, q-1)
$$

with $q$ the size of the residue field of $K$. In [S] we claimed that $\Gamma(d) \leq$ $d\left((d+1)^{2 \tau+1}-1\right)$. Unfortunately, there is a simple but serious error in the final step of the proof in [S]: an appeal is made to Hensel's lemma in a situation where it might not apply $\left({ }^{1}\right)$. As a consequence, the main result of that paper is only proved $\left({ }^{2}\right)$ for $d=p^{\tau}$. In this paper we present a modification of the arguments in $[\mathrm{S}]$, obtaining a bound for all $d$ :

Theorem A. $\Gamma(d) \leq d\left(p^{3 \tau} m^{2}\right)^{2 \tau+1}$.

In particular, $\Gamma(d) \leq d^{6 \tau+4}$.

2000 Mathematics Subject Classification: Primary 11D72, 11D88, 11E76.

Research supported in part by a fellowship from the David and Lucile Packard Foundation and a grant from the National Science Foundation.

$\left.{ }^{1}\right)$ The author discovered this error shortly after the publication of [S]. The error is cited in $[\mathrm{K}]$. The author's interest in this problem was recently rekindled by a conversation with David Leep.

$\left({ }^{2}\right)$ In $[\mathrm{R}]$ it is shown that the methods of [S] extend to the case $(d, q-1)=1$ giving the same bound for $\Gamma(d)$ as claimed in $[\mathrm{S}]$. 
We prove Theorem A by demonstrating that the existence of a non-trivial solution in $K$ to an equation as in (1) can be deduced from the existence of a non-trivial solution in $K$ to a certain system of additive equations of degree $m$. So we are naturally led to investigate the solvability of systems

$$
a_{1 j} x_{1}^{m}+\cdots+a_{s j} x_{s}^{m}=0, \quad j=1, \ldots, R,
$$

with coefficients $a_{i j}$ in $K$.

If we let $\Gamma(R, m)$ be the smallest integer such that any system as in (2) has a solution $0 \neq\left(x_{1}, \ldots, x_{s}\right) \in K^{s}$ whenever $s>\Gamma(R, m)$, then

Theorem B ([BG, Theorem 3]). $\Gamma(R, m) \leq R^{2} m^{2}$.

To be precise, Brüdern and Godinho only state and prove their theorem for the case $K=\mathbb{Q}_{p}$. However, it is easily checked that all the results used in that proof carry over to any $K$. For the interested reader as well as for a semblance of completeness, in Section 3 we indicate how to carry over these arguments.

The connection between Theorems A and B is the observation that $\Gamma(d) \leq d\left(p^{\tau} \Gamma\left(p^{\tau}, m\right)\right)^{2 \tau+1}$ (compare Lemmas 1 and 2).

2. Reducing Theorem A to Theorem B. We let $\mathcal{O}$ denote the integer ring of the local field $K$, fix a uniformizer $\pi \in \mathcal{O}$, and let $k=\mathcal{O} /(\pi)$ be the residue field of $K$. We denote by $\Gamma_{1}(d)$ the smallest integer such that any additive equation as in (1) with each $a_{i} \in \mathcal{O}^{\times}$has a non-trivial solution in $K$. For each positive integer $r$ we denote by $\Phi(d, r)$ the smallest integer such that if $s>\Phi(d, r)$ then any congruence equation

$$
a_{1} x_{1}^{d}+\cdots+a_{s} x_{s}^{d} \equiv 0\left(\bmod p^{r}\right), \quad a_{i} \in \mathcal{O},
$$

has a solution $\left(x_{1}, \ldots, x_{s}\right) \in \mathcal{O}^{s}$ with some $x_{j} \in \mathcal{O}^{\times}$. Of course, these notations only make sense provided the integers in question exist.

Lemma 1. Let $d=p^{\tau} m$ with $p \nmid m$. If $\Phi(d, 1)$ exists then so do $\Gamma(d)$, $\Gamma_{1}(d)$, and $\Phi(d, r)($ any $r>0)$. In particular,

(i) $\Phi(d, r+1) \leq \Phi(d, 1) \Phi(d, r)$.

(ii) $\Gamma_{1}(d) \leq \Phi(d, 2 \tau+1)$.

(iii) $\Gamma(d) \leq d \Gamma_{1}(d)$.

(iv) $\Gamma(d) \leq d \Phi(d, 1)^{2 \tau+1}$.

This is just Lemma 1 of [S]. In any event, these reductions are elementary and involve only standard techniques. For example, (ii) is a simple consequence of a version of Hensel's lemma.

Lemma 2. Let $d=p^{\tau} m$ with $p \nmid m$. If $\Gamma\left(p^{\tau}, m\right)$ exists, then so does $\Phi(d, 1)$ and

$$
\Phi(d, 1) \leq p^{\tau} \Gamma\left(p^{\tau}, m\right) .
$$


Proof. Assume that $\Gamma\left(p^{\tau}, m\right)$ exists. Suppose $a_{1} x_{1}^{d}+\cdots+a_{s} x_{s}^{d}$ to be as in (3). Writing each $a_{i}$ as $a_{i}=\pi^{r_{i}+p^{\tau} t_{i}} b_{i}$ with $0 \leq r_{i}<p^{\tau}$ and $b_{i} \in \mathcal{O}^{\times}$, we see that if $s>p^{\tau} \Gamma\left(p^{\tau}, m\right)$, then at least $\Gamma\left(p^{\tau}, m\right)+1$ of the $r_{i}$ 's are the same. Let $N=\Gamma\left(p^{\tau}, m\right)+1$. Relabeling our variables if necessary, we can assume that $r_{1}=\cdots=r_{N}$. It follows that the congruence (3) with $r=1$ has a solution $\left(x_{1}, \ldots, x_{s}\right) \in \mathcal{O}^{s}$ with some $x_{i} \in \mathcal{O}^{\times}$if the congruence

$$
\pi^{p^{\tau} t_{1}} b_{1} x_{1}^{d}+\cdots+\pi^{p^{\tau} t_{N}} b_{N} x_{N}^{d} \equiv 0(\bmod p)
$$

has a solution $\left(x_{1}, \ldots, x_{N}\right) \in \mathcal{O}^{N}$ with some $x_{i} \in \mathcal{O}^{\times}$.

For $\alpha \in k$ we define $u_{\alpha} \in \mathcal{O}$ as follows. If $\alpha=0$ then $u_{\alpha}=0$, but if $\alpha \neq 0$ then $u_{\alpha}$ is the unique element in $\mathcal{O}$ such that $u_{\alpha}^{q-1}=1$ and $u_{\alpha} \bmod \pi=\alpha$, where $q$ is the order of $k$. The existence and uniqueness of $u_{\alpha}$ is an easy consequence of Hensel's lemma. The association $\alpha \mapsto u_{\alpha}$ is multiplicative: $u_{\alpha} u_{\beta}=u_{\alpha \beta}$. We let $\mathbf{T}=\left\{u_{\alpha}: \alpha \in k\right\}$. Then for any $r \geq 0$ the map $\mathbf{T} \rightarrow \mathbf{T}$, $u \mapsto u^{p^{r}}$, is a bijection. Also, since $\mathbf{T}$ is a complete set of representatives for the residue field $k$, each $x \in \mathcal{O}$ can be uniquely written as $x=\sum_{n=0}^{\infty} v_{n} \pi^{n}$, $v_{n} \in \mathbf{T}$.

Writing $b_{i}=\sum_{n=0}^{\infty} v_{n, i} \pi^{n}, v_{n, i} \in \mathbf{T}$, we let $h_{n, i} \in \mathbf{T}$ be the unique element such that $h_{n, i}^{p^{\tau}}=v_{n, i}$. Putting $f=\left[e / p^{\tau}\right]$ where $e$ is defined by $(p)=\left(\pi^{e}\right)$, we then let

$$
c_{i, j}=\sum_{n=0}^{f} h_{p^{\tau} n+j, i} \pi^{n}, \quad j=0, \ldots, p^{\tau}-1 .
$$

Since

$$
c_{i, j}^{p^{\tau}} \equiv \sum_{n=0}^{f} h_{p^{\tau} n+j, i}^{p^{\tau}} \pi^{p^{\tau} n} \equiv \sum_{n=0}^{f} v_{p^{\tau} n+j, i} \pi^{p^{\tau} n}(\bmod p),
$$

we have

$$
b_{i} \equiv \sum_{j=0}^{p^{\tau}-1} \pi^{j} c_{i, j}^{p^{\tau}}(\bmod p) .
$$

From this we see that the congruence (4) has a solution of the desired type if the system of congruence equations

$$
\left(\pi^{t_{1}} c_{1, j}\right)^{p^{\tau}} x_{1}^{d}+\cdots+\left(\pi^{t_{N}} c_{N, j}\right)^{p^{\tau}} x_{N}^{d} \equiv 0(\bmod p), \quad j=0, \ldots, p^{\tau}-1,
$$

has a solution $\left(x_{1}, \ldots, x_{N}\right) \in \mathcal{O}^{N}$ with some $x_{i} \in \mathcal{O}^{\times}$. But, since $d=p^{\tau} m$,

$$
\left(\sum_{i=1}^{N} \pi^{t_{i}} c_{i, j} x_{i}^{m}\right)^{p^{\tau}} \equiv \sum_{i=1}^{N}\left(\pi^{t_{i}} c_{i, j}\right)^{p^{\tau}} x_{i}^{d}(\bmod p) .
$$

Therefore, the system (5) has a solution of the sought-for type if the system

$$
\pi^{t_{1}} c_{1, j} x_{1}^{m}+\cdots+\pi^{t_{N}} c_{N, j} x_{N}^{m} \equiv 0(\bmod p), \quad j=0, \ldots, p^{\tau}-1,
$$


has such a solution. And finally we note that (6) has such a solution if the system of equations

$$
\pi^{t_{1}} c_{1, j} x_{1}^{m}+\cdots+\pi^{t_{N}} c_{N, j} x_{N}^{m}=0, \quad j=0, \ldots, p^{\tau}-1,
$$

has a non-trivial solution in $K$ (for by homogeneity such a non-trivial solution $\left(x_{1}, \ldots, x_{N}\right)$ can always be scaled so that each $x_{i}$ is in $\mathcal{O}$ and not all the $x_{i}$ 's are divisible by $\left.\pi\right)$. Since $N>\Gamma\left(p^{\tau}, m\right),(7)$ has a non-trivial solution in $K$.

Assuming Theorem B, we obtain Theorem A by combining part (iv) of Lemma 1 with Lemma 2.

3. Remarks on the proof of Theorem B. We begin by noting that if $R=1$ then the bound in Theorem B follows from part (i) of Lemma 1 together with the observation that since $p \nmid m$, the theorem of ChevalleyWarning together with Hensel's lemma implies that $\Gamma_{1}(m) \leq m$.

Next we indicate how to obtain the same bound on $\Gamma(R, m)$ for a general $K$ as that given in [BG, Theorem 3] for $K=\mathbb{Q}_{p}$ (when $R \geq 2$ this bound is slightly better than that stated in Theorem B). More precisely, we explain how to modify the statements of the results used in the proof in [BG] so that they apply to the general situation, that is, to the situation where "systems" are systems of equations or congruences with coefficients in $\mathcal{O}$ and "solutions" are solutions with entries in $\mathcal{O}$. We use without explanation some of the terminology and notation from [BG].

First we note that the notions of $p$-normalized systems of additive equations and $p$-equivalence have immediate generalizations to $\pi$-normalized systems and $\pi$-equivalence: one merely replaces $p$ with $\pi$ in the definition. Similarly, $p$ must be replaced by $\pi$ in the definition of the level of a variable. Then all the results from [DL] quoted in [BG] continue to hold for $\pi$-normalized systems; the proofs are exactly the same. In particular, [BG, Lemma 1] holds with $p$ replaced by $\pi$ and "integer coefficients" meaning coefficients in $\mathcal{O}$.

Next we note that the result from [LPW] quoted in $[\mathrm{BG}]$ also holds for $\pi$-normalized systems. In [LPW] this result is deduced by reducing the system modulo $p$ and applying a combinatorial result about matrices over fields. Since this combinatorial result is proved in [LPW] for any field (and so for $k$ ) the same argument applies to the reduction modulo $\pi$ of a $\pi$ normalized system. Thus [BG, Lemma 2] holds with $p$ replaced by $\pi$.

We also note that the version of Hensel's lemma quoted in [BG, Lemma 3] also holds over $K$ without change, but in the definition of a non-singular solution of a system of congruences such as [BG, (10)], $p$ gets replaced by $\pi$ (i.e., the condition is $x_{i_{1}} \cdots x_{i_{R}} \operatorname{det}\left(\mathbf{a}_{i_{1}} \ldots \mathbf{a}_{i_{R}}\right) \not \equiv 0(\bmod \pi)$ ).

Similarly, [BG, Lemma 4] holds with the $p$ in the congruence [BG, (12)] replaced by $\pi$, the $p-1$ in the definition of $\delta$ replaced by $q-1$ with $q$ the 
order of the residue field $k$ of $K$, and with the $c_{i j}$ 's allowed to be in $\mathcal{O}$; this is still the theorem of Chevalley-Warning. It then follows that [BG, Lemma 5] holds with $p$ replaced by $\pi$; the same proof works.

Combining the modified versions of [BG, Lemmas 1-5] then implies that $\Gamma(R, m) \leq R m(R(m, q-1)-R+2)$, where $q$ is the order of the residue field of $K$.

A final remark. Finally, we note that an elementary argument of Leep and Schmidt (cf. [LS, (2.11)]) shows that a system of $R$ equations as in (1) has a non-trivial solution in $K$ provided $s>(\Gamma(d)+1)^{R}$, so in particular if $s>\left(d^{6 \tau+4}+1\right)^{R}$. However, it should be possible to adapt the methods of this paper to prove that there is an integer $c$ such that a non-trivial solution exists if $s>(R d)^{c \tau}$.

\section{References}

[B] B. J. Birch, Diagonal equations over p-adic fields, Acta Arith. 9 (1964), 291-300.

[BG] J. Brüdern and H. Godinho, On Artin's conjecture I. Systems of diagonal forms, Bull. London Math. Soc. 31 (1999), 305-313.

[DL] H. Davenport and D. J. Lewis, Simultaneous equations of additive type, Philos. Trans. Roy. Soc. London Ser. A 264 (1969), 557-595.

[K] M. Knapp, Systems of diagonal equations over $p$-adic fields, J. London Math. Soc. (2) 63 (2001), 257-267.

[LS] D. Leep and W. Schmidt, Systems of homogeneous equations, Invent. Math. 71 (1983), 539-549.

[LPW] L. Low, J. Pitman, and A. Wolff, Simultaneous diagonal congruences, J. Number Theory 29 (1988), 31-59.

[R] A. Rangachev, On the solvability of $\mathfrak{p}$-adic diagonal equations, preprint, 2004.

[S] C. Skinner, Solvability of $\mathfrak{p}$-adic diagonal equations, Acta Arith. 75 (1996), 251258.

Department of Mathematics

University of Michigan

Ann Arbor, MI 48109-1043, U.S.A.

E-mail: cskinner@umich.edu 